\title{
Regulation of cotton fiber elongation by xyloglucan endotransglycosylase/hydrolase genes
}

\author{
M.Y. Shao ${ }^{1}$, X.D. Wang ${ }^{1}$, M. Ni ${ }^{1}$, N. Bibi ${ }^{1,2}$, S.N. Yuan ${ }^{1}$, W. Malik ${ }^{1,3}$, \\ H.P. Zhang' ${ }^{1}$ Y.X. Liu ${ }^{1}$ and S.J. Hua ${ }^{1}$ \\ ${ }^{1}$ Department of Agronomy, Zhejiang University, Hangzhou, P.R. China \\ ${ }^{2}$ Plant Breeding and Genetic Division, \\ Nuclear Institute for Agriculture and Biology, Faisalabad, Pakistan \\ ${ }^{3}$ Department of Plant Breeding and Genetics, \\ College of Agriculture, Bahauddin Zakariya University, Multan, Pakistan \\ Corresponding author: X.D. Wang \\ E-mail: xdwang@zju.edu.cn
}

Genet. Mol. Res. 10 (4): 3771-3782 (2011)

Received May 19, 2011

Accepted October 7, 2011

Published October 27, 2011

DOI http://dx.doi.org/10.4238/2011.October.27.1

\begin{abstract}
Ligon lintless mutant (li1li1) with super-short fibers (5-8 $\mathrm{mm}$ in length) and its wild type (Li1Li1) with normal fibers (30 $\mathrm{mm}$ in length) were used to study the function of xyloglucan endotransglycosylase/hydrolase (XTH) genes during fiber elongation in cotton. Wild-type cotton attained the fiber elongation stage earlier (5 days post-anthesis, DPA), than the Ligon lintless mutant (12 DPA) with a higher fiber elongation velocity of about $1.76 \mathrm{~mm} /$ day. Xyloglucan contents in Ligon lintless mutant fibers were 5-fold higher than the wild type during 9-15 DPA. It was also observed that the activity of XTH in wild-type cotton fibers was about 2-fold higher than that of the Ligon lintless mutant with a peak at 12 DPA. DNA blot analysis indicated that the $X T H$ gene in the Ligon lintless mutant and its wild type belonged to a multiple allelic series. However, RNA blot analysis and quantitative real-time PCR exhibited an earlier expression (10 DPA) of XTH in wild type as compared to delayed (15 DPA) expression in the Ligon lintless mutant. The study also revealed that 9-15 DPA might be a key phase
\end{abstract}


for upregulation of fiber elongation via increasing XTH activity. Higher $\mathrm{XTH}$ activity can cleave down the xyloglucan-cellulose chains thus loosening fiber cell wall and promoting fiber cell elongation in wild type as compared to its mutant.

Key words: Fiber elongation; Fiber length; Ligon lintless mutant; Xyloglucan endotransglycosylase/hydrolase; Xyloglucan

\section{INTRODUCTION}

Cotton fiber, a unicellular structure, arises from single epidermal cells of developing seeds without undergoing cell division. Fiber development usually consists of four overlapping stages: initiation, elongation, secondary wall deposition and maturation (Basra and Malik, 1984). In cotton, the elongation phase of fiber development is long enough to result in longer $(>30 \mathrm{~mm})$ fiber length and is thus regarded as a model system for studying the mechanisms of plant cell elongation (Kim and Triplett, 2001).

The primary cell wall of the plant cell has a dual role during expansion: to sustain the large mechanical forces caused by cell turgor, and to permit controlled polymer extension generating more space for protoplast enlargement (Cosgrove, 2001). Plant cell wall comprised of cellulose and hemicellulose (Brett and Waldron, 1996; Pauly et al., 1999). Hemi-cellulose fraction is further composed of xyloglucan molecules that cross link the cellulose microfibrills through non-covalent hydrogen bonds (Cosgrove, 2001; Vissenberg et al., 2005). These noncovalent bonds are disrupted by the action of (Fry et al., 1992) and xyloglucan endotransglycosylase/hydrolases (XTHs) and expansin thus increasing cell wall plasticity and finally fiber elongation (Ruan et al., 1998; Smart et al., 1998).

Xyloglucan endotransglycosylase/hydrolases (XTHs) are actually enzymes that cleave donor xyloglucan chains and rejoin the newly formed ends to the non-reducing terminus of an available acceptor xyloglucan chain or oligosaccharide/water. Since XTHs induce new xyloglucans under certain conditions and also cause hydrolytic cleavage of xyloglucan, thought to be the enzymes that loosen the cell walls to lead cell expansion and elongation (Cosgrove, 2005; Nishitani and Vissenberg, 2007). The relationship of XTH activity and cell elongation has been reported in elongating cotton fiber (Shimizu et al., 1997; Michailidis et al., 2009; Lee et al., 2010) as well as in other crops like maize, cucumber, tobacco, potato, soybean, rice and Arabidopsis (Silva et al., 1994; Potter and Fry, 1994; Vissenberg et al., 2000; Michailidis et al., 2009).

Similarly, expansin genes and $\beta$-1, 4-glucanase genes are expressed strongly during fiber elongation and regulate the extension of fiber cell wall (Hasegawa et al., 1994; Shimizu et al., 1997). $\mathrm{H}^{+}$-ATPase, sucrose synthase, $\mathrm{K}^{+}$transporters and plasmodesmata switch control of the transportation of sucrose and $\mathrm{K}^{+}$to fiber cell vacuole thus bringing changes in cell osmotic pressure and finally resulting in cell elongation (Ruan et al., 2001). Fiber elongation seems to be associated with changes in cell turgor pressure (Ruan et al., 2000) and achieved largely through the influx of water driven by a relatively high concentration of osmoticum within a cell (Cosgrove, 1997). The accumulation of osmoticum in fibers may be coupled with the transmembrane proton gradient, because the plasma membrane H1 - ATPase gene is expressed strongly during the rapid phase of fiber elongation (Smart et al., 1998). This H1 pump thus 
acidifies the apoplast for cell wall loosening (Cosgrove, 1997).

Normally fiber elongation begins on the day of anthesis and continues for 20-30 days, during which time cotton fibers grow to 3-6 cm long depending on the cultivar (Ryser, 1999) and attain $30 \mathrm{~mm} 20$ days post-anthesis (DPA) (Schubert et al., 1973; Basra and Malik, 1984). However, in some mutants, fibers do not grow to more than $10 \mathrm{~mm}$ and have fuzzy seeds (short lint fibers, Ligon lintless), a trait controlled by a completely dominant gene, $L i_{2}$ (Narbuth and Kohel, 1990), located on chromosome 18 (Kohel et al., 2002). Proper folding of the HSP 90 is necessary for fiber elongation and its regulation may be affected by the $L i_{1}$ mutation (Zhao et al., 2010) in Ligon lintless as compared to its wild type fibers.

Although there are many studies on cotton fiber elongation the mechanism is still unrevealed. The present research project was planned to utilize Ligon lintless $\left(l i_{1} l i_{1}\right)$ with supershort fibers (only about 5-8 $\mathrm{mm}$ in length) and its wild type with $30 \mathrm{~mm}$ long fibers as experimental material to broaden the understanding of the involvement of $X T H$ in fiber elongation.

\section{MATERIAL AND METHODS}

\section{Plant materials}

Two lines of allotetraploid cotton (Gossypium hirsutum L.), a wild type $\left(L i_{1} L i_{1}\right)$ with 30 $\mathrm{mm}$ long fiber and the Ligon Lintless $\left(l i_{1} l i_{1}\right)$ mutant with 5-8 $\mathrm{mm}$ long fiber (Figure 1) were grown at the Experiment Farm, Zhejiang University, Hangzhou, China. Flowers were tagged on the day of anthesis and the collection of seeds with developing fibers was done at different stages.

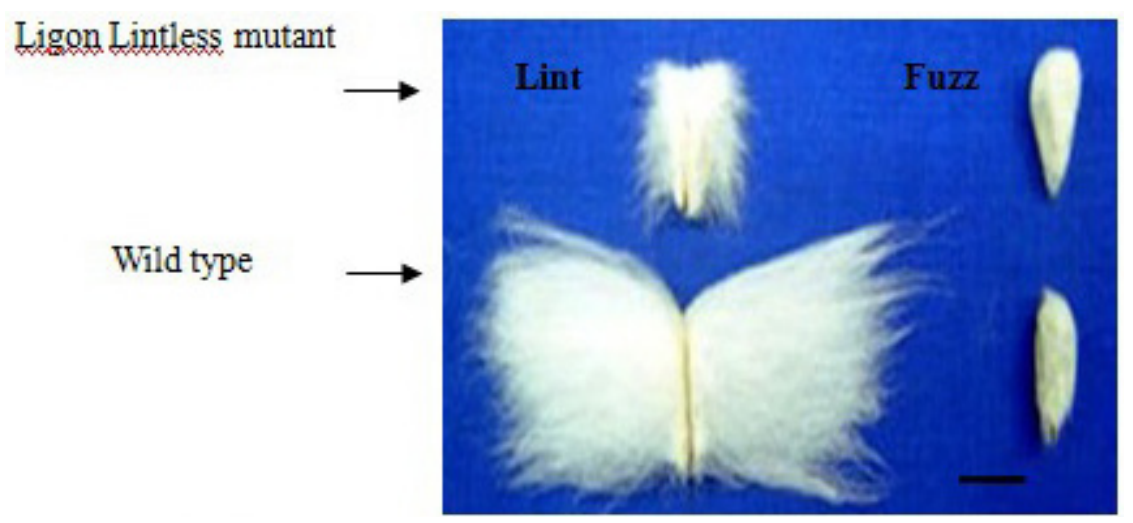

Figure 1. Seed and fiber morphology of Ligon lintless mutant and its wild type (scale bar: $10 \mathrm{~mm}$ ).

\section{Fiber length}

Three healthy bolls (10 seeds/boll) from various phases $(3-10,12,14,19,23,28,33$, and 40 DPA) of post-anthesis were collected. To measure the fiber length, 3-5 DPA cotton seeds were treated with boiling water for about two minutes and then observed under a stereo microscope (C3 model, 0.1 ocular ruler). The fiber length of 5-40 DPA cotton seeds was measured manually after treating with boiling water for about five minutes. Fiber elongation velocity was calculated using the following formula: 
Fiber elongation velocity $=($ fiber length at $\max$ DPA - fiber length at $\min$ DPA $) /(\max$ DPA- min DPA)

\section{Xyloglucan contents}

To quantify the XG contents, cotton fibers were collected at $6,9,12,15,18,21,25$, $30 \mathrm{DPA}$ seeds and dried at $80^{\circ} \mathrm{C}$ in an oven. About $10 \mathrm{~g}$ of dried fibers were then milled in 10 $\mathrm{mL}$ water, followed by the addition of $90 \mathrm{~mL} 2 \mathrm{M} \mathrm{NaOH} / 0.05 \% \mathrm{NaBH}_{4}$ and boiled for $1 \mathrm{~h}$ at $100^{\circ} \mathrm{C}$. After centrifugation for $10 \mathrm{~min}$ at $1400 \mathrm{~g}$, supernatant was collected in another set of tubes and $200 \mathrm{~mL}$ acetic acid/ethanol (1:10) added, kept for $30 \mathrm{~min}$ at room temperature and again centrifuged to yield an XG pellet (Edwards et al., 1985).

For purification, $\mathrm{XG}$ was dissolved in a solution of Fehling reagent $\mathrm{B}(35 \%$ $\left.\mathrm{KNaC}_{4} \mathrm{H}_{4} \mathrm{O}_{6} \cdot 4 \mathrm{H}_{2} \mathrm{O}, 25 \% \mathrm{KOH}\right)$ with water in $1: 8$ ratio respectively, boiled for $30 \mathrm{~min}$ at $100^{\circ} \mathrm{C}$, cooled at room temperature and finally followed by the addition of $10 \mathrm{~mL}$ Fehling reagent $\mathrm{A}\left[7.5 \% \mathrm{CuSO}_{4}\right]$. Centrifugation was done for $10 \mathrm{~min}(1400 \mathrm{~g})$, the supernatant decanted off and the pellet dissolved in $20 \%$ acetic acid solution. XG was again precipitated by adding a double volume of absolute ethanol, centrifuged and the pellet finally dissolved in $50 \%$ ethanol (containing 5\% EDTA). After centrifugation, the pellet was washed with 50\% ethanol and freeze-dried in a Modul Yod-230 (America, Thermo savant) at $-80^{\circ} \mathrm{C}$ and weighed. The $\mathrm{XG}$ content was then calculated as

$$
\text { Xyloglucan content }(\%)=X G \text { dry weight/total fiber weight*100\%. }
$$

\section{Xyloglucan endotransglycosylase/hydrolase (XTH) activity}

XTH activity was determined by the colorimetric method (Sulová et al., 1995; Henriksson et al., 2003). The method comprised four steps: first XG was extracted from ground tamarind (Tamarindus indica) kernels (Edwards et al., 1985); second Xyloglucan-derived oligosaccharides (XGOs) were prepared according to Henriksson et al. (2003); third XTH was extracted from cotton fiber (Henriksson et al., 2003) and forth colorimetric analysis was conducted on XTH activity. The optical density of the samples was measured at $620 \mathrm{~nm}$ against the blank. The activity of XTH was calculated according to the following formula described by Sulová et al. (1995).

$$
\mathrm{XTHA}=100(\mathrm{~A} 1-\mathrm{A} 2) / \mathrm{A} 1-100(\mathrm{~A} 3-\mathrm{A} 4) / \mathrm{A} 3
$$

\begin{tabular}{|c|c|c|c|c|}
\hline Treatments & $\mathrm{XG}$ & XTH & $\mathrm{XGO}_{\mathrm{s}}$ & buffer \\
\hline Blank & - & + & + & + \\
\hline$A_{1}$ & + & - & + & + \\
\hline$A_{2}$ & + & + & + & + \\
\hline $\mathrm{A}_{3}^{2}$ & + & - & - & + \\
\hline $\mathrm{A}_{4}^{3}$ & + & + & - & + \\
\hline
\end{tabular}

XTHA is the activity of XTH, which is measured in $\mathrm{mg} /$ protein (Table 1). 


\section{DNA and RNA blot hybridization}

Primers ( $X T H$-forward: 5'-GATTTTATCTTGTGTTGTTACACTTTC-3' and XTH-reverse: 5'-GATATTGGTTTGAACCGTATATGGC-3') were synthesized from the already available gene sequence (XTH1) in the database. A 343-bp fragment of the XTH coding region was then amplified using these primers and used as a hybridization probe (Shimizu et al., 1997). Cotton genomic DNA isolated from the Ligon lintless mutant and its wild type were digested with restriction endonucleases EcoRI and HindW in separate reactions. The digested DNA $(10 \mu \mathrm{g})$ was separated on $0.8 \%(\mathrm{w} / \mathrm{v})$ agarose gel. DNA fragments from agarose gel were then transferred onto nylon membranes (Hybond $\mathrm{N}^{+}$, Amersham, USA) in 20X SSC by capillary blotting and hybridized under high stringency conditions by using a DIG-labeled probe and the detection kit I (Roche, Germany).

Total RNA from cotton fiber (stored at $-70^{\circ} \mathrm{C}$ ) was extracted according to the method described by Xu et al. (2002), separated ( $5 \mu \mathrm{g})$ on $0.8 \%$ (w/v) formaldehyde gel and finally blotted onto nylon membrane (Hybond $\mathrm{N}^{+}$, Amersham) in 20X SSC by capillary blotting. A DIG-labeled XTH gene (Shimizu et al., 1997) was used as a probe to detect the RNA.

\section{Quantitative real-time PCR}

To monitor the expression of $X T H$, quantitative real-time PCR was performed by using the alpha-tubulin gene (TubA4) as a normalizer. Specific primers against $X T H$ were synthesized (Table 2) from the already available sequences of Gossypium hirsutum XTHs mRNA (Accession number D88413) in NCBI.

Table 2. Specific primers used for quantitative real-time PCR analysis.
\begin{tabular}{lll}
\hline Gene & Forward primer $\left(5^{\prime}-3^{\prime}\right)$ & Reverse primer $\left(5^{\prime}-3^{\prime}\right)$ \\
\hline$X T H$ & AGCCATCCAACTTGTTCTCG & GCTCATCTCGCACAGCATC \\
TubA4 & CTCGCTGCCCTGGAAA & CACAGTAGCAAACGGATAACG \\
\hline
\end{tabular}

A two-step qRT-PCR kit with SYBR ${ }^{\circledR}$ Green (Invitrogen, Carlsbad, CA) was used for detecting the $X T H$ expression levels. The reaction mixture (three replications for each sample) contained $10 \mu \mathrm{L}$ Platinum SYBR Green qPCR SuperMix-UDG, $0.4 \mu \mathrm{L}$ of $10 \mu \mathrm{M}$ forward and reverse primers each, $1 \mu \mathrm{L}$ cDNA and DEPC-treated water up to $20 \mu \mathrm{L}$. The relative expression levels of all the samples were analyzed according to the method recommended in User Bulletin No. 2 for the ABI PRISM 7700 Sequence Detection System (Applied Biosystems, Foster City, CA, USA). The $\Delta \mathrm{Ct}$ and $\Delta \Delta \mathrm{Ct}$ for XTH gene and TubA4 were calculated from threshold cycles (Ct value) as:

$\Delta \mathrm{Ct}$ value of the sample $=\mathrm{Ct}$ value of target gene $-\mathrm{Ct}$ value of normalizer TubA4.

The $\Delta \mathrm{Ct}$ value of the calibrator (the sample with the highest $\Delta \mathrm{Ct}$ value) was subtracted from every other sample to produce the $\Delta \Delta \mathrm{Ct}$ value and $2^{-\Delta \Delta \mathrm{Ct}}$ was taken for every sample as the relative expression level.

\section{RESULTS}

\section{Fiber length variance}

The analysis of fiber length measurement revealed superior fiber length $(30 \mathrm{~mm})$ in 
the wild type cotton over Ligon lintless $(<10 \mathrm{~mm})$ fiber. The fiber elongation phase was initiated in wild type (5 DPA) (Figure 2) prior to its Ligon lintless mutant (12 DPA).

Similarly, duration of fiber development was divided into initiation, elongation and termination phase with a prolonged (5-19 DPA) period of fiber elongation in the wild type, unlike the Ligon Lintless mutant (12-19 DPA only).

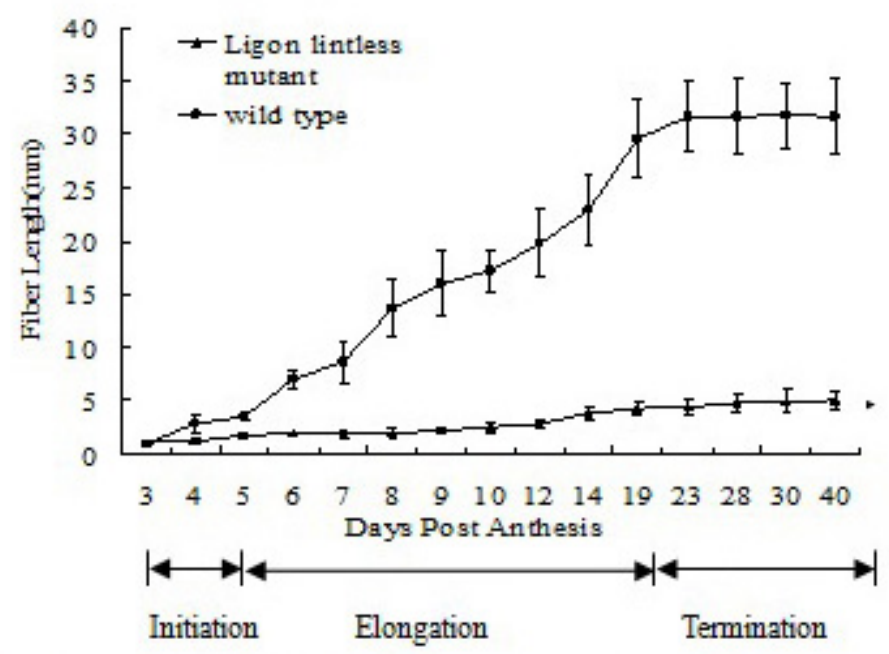

Figure 2. Dynamics of Fiber Elongation in Ligon lintless and wild type cotton.

\section{Contents of xyloglucan (XG)}

The xyloglucan $(\mathrm{XG})$ contents in the Ligon lintless mutant were significantly higher ( $\mathrm{P}$ $<0.01$ ) than that of the wild type during all stages of fiber development (Figure 3). However, the XG contents started decreasing from 9 DPA, remained constant up to 12 DPA and declined sharply from 15 DPA to the end of the fiber elongation stage in Ligon lintless cotton. On the other hand, XG contents remained constant from 9-18 DPA (fiber elongation phase) in the wild type. This evidence supports the fact that the wild type has an earlier and longer fiber elongation phase (5-19 DPA) than Ligon lintless (12-19 DPA). With subsequent developmental phases, the XG content of both cultivars declined by a similar amount at the end of fiber elongation stage in both cultivars suggesting XG involvement during fiber elongation.

Since XG is an important component in the fiber cell wall, the higher XG contents in the mutant fiber might make its cell wall harder and then limit fiber cell elongation.

\section{Xyloglucan endotransglycosylase/hydrolase (XTH) activity}

The trends of XTH activity varied between the Ligon lintless mutant and its wild type during the fiber elongation phase (5-19 DPA) while in later stages (21-30 DPA) the behavior of both genotypes was similar. When compared, the XTH activity of the wild type was found to be higher than the Ligon lintless mutant in all fiber-elongation stages (Figure 4). In the wild 


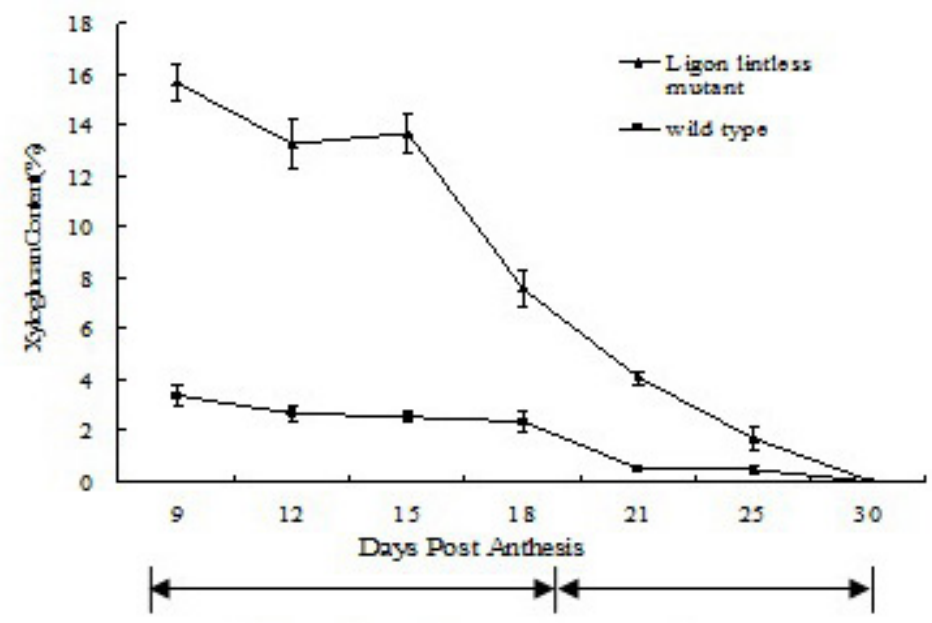

Elongation Phase Termination phase

Figure 3. Xyloglucan content of Ligon lintless mutant and wild type.

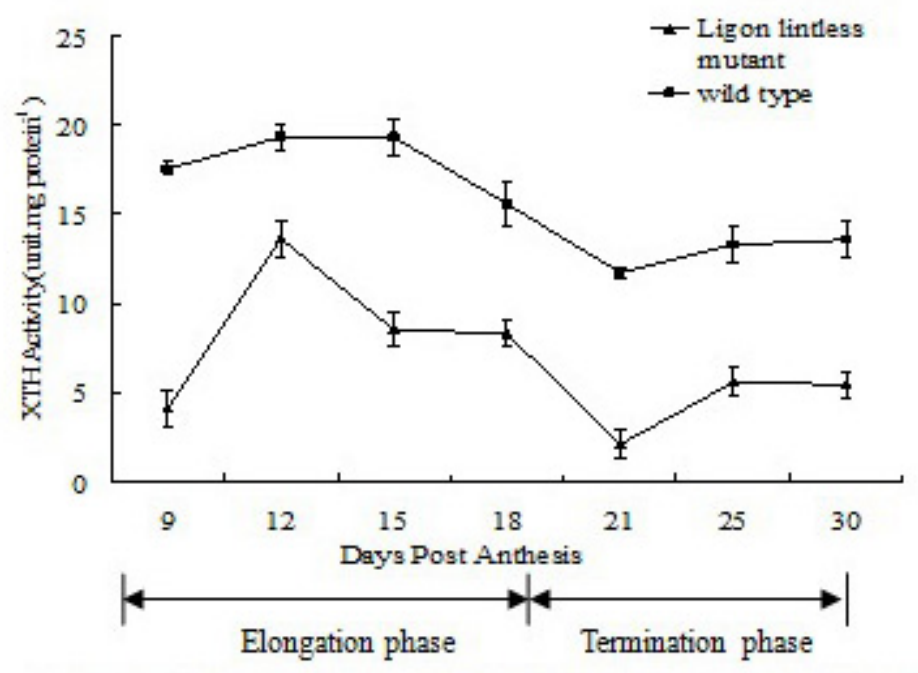

Figure 4. XTH activity of Ligon lintless and wild type cotton fiber.

type, XTH activity increased up to 12 DPA with a constant value up to 15 DPA, followed by a sharp decline from 15-21 DPA, whereas the Ligon lintless mutant exhibited a cyclic pattern of alternate increases and decreases in XTH activity.

\section{Genomic Southern blot analysis}

To study the copy number of XTH in the Ligon lintless mutant and its wild type, DNA 
blotting analysis was performed using EcoRI and HindIII restriction endonucleases in separate reactions. Three bands were observed at the EcoRI digest, and four bands were detected at the HindIII digest thus suggesting the presence of multiple copies of the same gene (Figure 5).

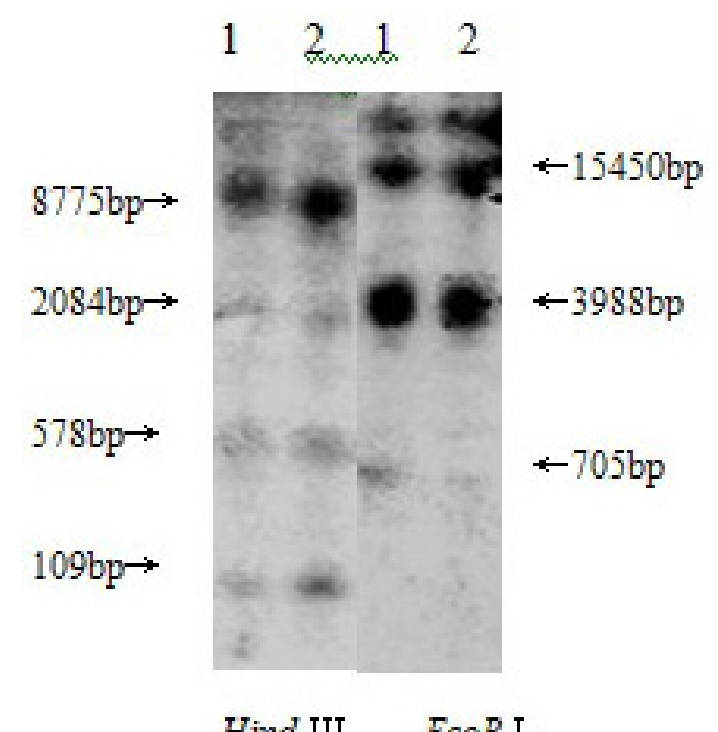

Figure 5. Southern blot hybridization from wild type (lane 1) and Ligon lintless mutant (lane 2) with probe of XTH gene. Each lane contains $15 \mu \mathrm{g}$ genomic DNA fragments.

\section{Northern blot analysis}

Northern hybridization exhibited a superior expression of $X T H$ in fiber samples of both the Ligon lintless mutant and its wild type as compared to their leaves (Figure 6a,b).

In addition, $X T H$ in fiber of the wild type had an earlier (10 DPA) and higher expression level as compared to that in fiber of the Ligon lintless mutant (15 DPA) during the fast fiber-elongation stage.

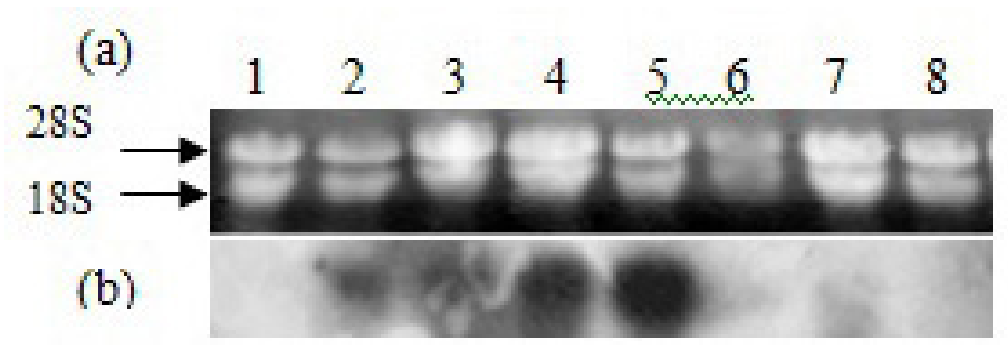

Figure 6. A. Agarose gel electrophoresis of RNA (15 $\mu \mathrm{g} / \mathrm{lane})$. B. Northern blot Hybridization probed by XTH gene. Lanes 1, 2 and 3 were 10,15 and 20 DPA fiber RNA from Ligon lintless mutant respectively; lanes 4, 5 and 6 were 10,15 and 20 DPA fiber RNA from wild-type; lane 7, 8 were leaf RNA from Ligon lintless mutant and wild-type respectively. 


\section{Quantitative real-time PCR}

Transcriptional analysis of $X T H$ was performed by quantitative real-time PCR method to monitor differences between the Ligon lintless mutant and its wild type during the fiberelongation phase.

Figure 7 shows that transcripts of $X T H$ were significantly higher $(\mathrm{P}<0.05)$ in the wild type as compared to the Ligon lintless mutant at all fiber developmental stages. However $X T H$ expression increased up to 15 DPA and then decreased suddenly (15-20 DPA) in the wild type with a less pronounced decrease in the Ligon lintless mutant. The findings were consistent with the results of XTH activity and to that of Northern hybridization. This might indicate that $X T H$ is an up-regulated gene during fiber elongation.

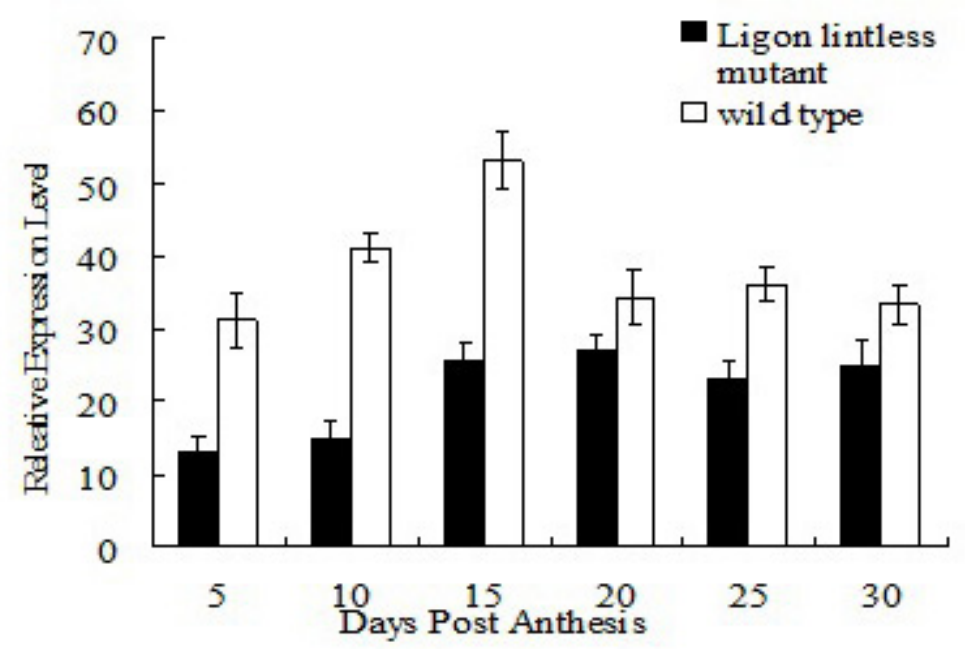

Figure 7. Quantitative real-time PCR using SYBR Green assays for quantitative analysis of $X T H$ mRNA expression levels in fiber of mutant and wild type. Data are the mean \pm SD from three replications.

\section{DISCUSSION}

Cotton is the mainstay of textile industries throughout the world and its quality depends on changes in the growth and developmental pattern of the fiber. Therefore, understanding the biological mechanisms that regulate fiber quality, especially fiber length, strength and micronare, is important for the long-term improvement of cotton.

Analysis of fiber length measurements revealed the Ligon lintless mutant to have a fiber length of less than $10 \mathrm{~mm}$, which is in accordance with the findings of An et al. (2010). The elongation of cotton fibers is driven by cell turgor pressure and is limited by the extensibility of the primary cell wall.

The cotton fiber elongation stage usually begins at 5 DPA and lasts up to 25 days postanthesis (Cosgrove, 2001) with a period of rapid cellular elongation of about 15-20 days (Lee et al., 2010). In the current study, initiation of fiber elongation was found to be delayed (12 DPA) in the Ligon lintless mutant as compared to its wild type (5 DPA). Similarly, the sharp 
fiber elongation phase was also very short (12-19 DPA) in Ligon lintless while it was long lasting (5-19 DPA) for wild type cotton with high fiber elongation velocity of about $1.76 \mathrm{~mm} /$ day, six-fold higher than the Ligon Lintless mutant. However after 19 DPA, the behavior of both cultivars became similar (constant phase) with no more fiber elongation.

Moreover, the saccharides test related the phenomenon of short cotton fiber with high contents of xyloglucan (XG Content) and lower XTH activity, thus confirming the study by Shimizu et al. (1997). In the current study as well, XG contents in the Ligon lintless mutant fiber were found to be much higher than that of wild type in all fiber's developmental stages. Since XG is an important component in the fiber cell wall, the higher XG content in the mutant fiber might make its cell wall harder and then limit fiber cell elongation. Conversely, the XTH activity in wild type fiber was higher than that of the Ligon lintless mutant in nearly all of the fiber-elongation stages. Since the XTH activity is low, there are not enough XTH molecules to cleave the chain of xyloglucan-cellulose and divide XG content to loosen the plant cell wall. This might be the reason for extremely short fibers in Ligon lintless cotton. Thus, on one hand, an increase of XTH activity breaks the linkage of cellulose-xyloglucan chains to lose fiber cell wall, and on the other hand, accelerates the disaggregation of xyloglucan to improve fiber cell turgor during the last stage of fiber cell elongation. Accordingly, it is presumed that the earlier and higher expression of $X T H$ in wild type might prevent xyloglucan from accumulating in cotton fiber thus promoting fiber elongation.

A large number of similar reports (Pritchard et al., 1993; Silva et al., 1994; Potter and Fry, 1994; Vissenberg et al., 2000) have indicated that XTH activity had a typical direct correlation with cell elongation which is in agreement with our findings. Thus fiber elongation of the Ligon lintless mutant (12-19 DPA) in the current study might be caused by the increase of XTH activity (which had a peak at 12DPA) and the decrease of xyloglucan content in cotton fiber at this particular stage.

DNA digestion with EcoRI and HindIII produced three and four fragments in both the wild type and the Ligon lintless mutant, suggesting XTH belonged to a multiple allelic series. A number of other reports from Gossypium (Shimizu et al., 1997; Michailidis et al., 2009; Lee et al., 2010) proved the multigenic nature of XTH. Similarly, high expression of $X T H$ has been detected in many elongating plant parts, for example, barley leaves (Schümann et al., 1997), pine epicotyls (Barrachina et al., 1998), maize roots (Pritchard et al., 1993), soybean stems (Potter and Fry, 1994), the elongation zone of Arabidopsis roots (Vissenberg et al., 2000), expanding cells and root hair start site (Verbelen et al., 2001), chickpea epicotyls (Romo et al., 2005) and elongating fiber cells (Georgios et al., 2009).

Corresponding RNA blot analysis confirmed that $X T H$ had a dominant expression in the fiber of the Ligon lintless mutant and its wild type whereas no transcript of $X T H$ was detected in leaves of either cultivar. Michailidis et al. (2009) also reported the highest levels of $G h X T H 1$ expression in elongating fibers while $G h X T H 2$ and $G h X T H 3$ were expressed at relatively low levels in fiber cells and have more pronounced expression in expanding petals $(G h X T H 2)$ leaves and roots $(G h X T H 3)$. XTH transcripts were also absent from the other cotton organs and mRNA levels of XTH were significantly higher at 10 DPA as compared to later stages of fiber elongation (25 DPA). So delayed and insufficient expression of XTH might be the reason for low XTH activity and high XG content in the Ligon lintless mutant, thus affecting the overall development of the fiber.

Lee et al. (2010) also detected that GhXTH1 strongly expressed at 10 DPA and had the highest expression at 15 DPA in field cotton fibers. Meanwhile, transgenic cotton plants 
that over-express $G h X T H 1$ had increased XTH activity and produced mature cotton fibers that were between 15 and $20 \%$ longer than wild-type cotton plants thus proving the involvement of $X T H$ in fiber elongation.

\section{CONCLUSIONS}

Combining our results and all the existing reports, we believe that $X T H$ plays an important role during the fiber cell elongation stage. In conclusion, the high XTH activity in cotton fiber may be useful for breaking the chains of cellulose-xyloglucan in the fiber cell wall and promoting fiber elongation. This study can form a basis for screening of the germplasms for improvement of fiber quality of cotton as $X T H$ is significantly upregulated in plants with longer fibers.

\section{ACKNOWLEDGMENTS}

Research partly supported by the Ministry of Agriculture of the People's Republic of China (\#2009ZX08009-061B).

\section{REFERENCES}

An C (2008). SNP Characterization and Genetic and Molecular Analysis of Mutants Affecting Fiber Development in Cotton. PhD thesis, Mississippi State University, Starkville. Available at [http://sun.library.msstate.edu/ETD-db/ theses/available/etd-03302008-191842]. Accessed....

Barrachina C and Lorences EP (1998). Xyloglucan endotransglycosylase activity in pine hypocotyls. Intracellular localization and relationship with endogenous growth. Physiol. Plant. 102: 55-60.

Basra AS and Malik CP (1984). Development of the cotton fiber. Int. Rev. Cytol. 89: 65-113.

Cosgrove DJ (1997). Assembly and enlargement of the primary cell wall in plants. Annu. Rev. Cell Dev. Biol. 13: 171-201.

Cosgrove DJ (2001). Wall structure and wall loosening. A look backwards and forwards. Plant Physiol. 125: 131-134.

Cosgrove DJ (2005). Growth of the plant cell wall. Nat. Rev. Mol. Cell Biol. 6: 850-861.

Edwards M, Dea ICM, Bulpin PV and Reid JSG (1985). Xyloglucan (amyloid) mobilization in the cotyledons of Tropaeolum majus L. seeds following fermination. Planta 163: 133-140.

Fry SC, Smith RC, Renwick KF, Martin DJ, et al. (1992). Xyloglucan endotransglycosylase, a new wall-loosening enzyme activity from plants. Biochem. J. 282: 821-828.

Hasegawa O, Aotsuka S, Takokoro F, Takenishi S, et al (1994). Random Sequencing of a cDNA Library Obtained from Cotton Fiber Cells. In: Kyoto Conference on Cellulose (Shiraishi N, ed.). The Cellulose Society of Japan, Kyoto, 87.

Henriksson H, Denman SE, Campuzano ID, Ademark P, et al. (2003). N-linked glycosylation of native and recombinant cauliflower xyloglucan endotransglycosylase 16A. Biochem. J. 375: 61-73.

Kim HJ and Triplett BA (2001). Cotton fiber growth in planta and in vitro. Models for plant cell elongation and cell wall biogenesis. Plant Physiol. 127: 1361-1366.

Kohel RJ, Stelly DM and Yu J (2002). Tests of six cotton (Gossypium hirsutum L.) mutants for association with aneuploids. J. Hered. 93: 130-132.

Lee J, Burns TH, Light G, Sun Y, et al. (2010). Xyloglucan endotransglycosylase/hydrolase genes in cotton and their role in fiber elongation. Planta 232: 1191-1205.

Maris A, Suslov D, Fry SC, Verbelen JP, et al. (2009). Enzymic characterization of two recombinant xyloglucan endotransglucosylase/hydrolase (XTH) proteins of Arabidopsis and their effect on root growth and cell wall extension. J. Exp. Bot. 60: 3959-3972.

Michailidis G, Argiriou A, Darzentas N and Tsaftaris A (2009). Analysis of xyloglucan endotransglycosylase/hydrolase (XTH) genes from allotetraploid (Gossypium hirsutum) cotton and its diploid progenitors expressed during fiber elongation. J. Plant Physiol. 166: 403-416.

Narbuth EV and Kohel RJ (1990). Inheritance and linkage analysis of a new fiber mutant in cotton. J. Hered. 81: 131-133. Nishitani K and Vissenberg K (2007). Roles of the XTH Family in the Expanding Cell. Springer, Berlin. 
Pauly M, Albersheim P, Darvill A and York WS (1999). Molecular domains of the cellulose/xyloglucan network in the cell walls of higher plants. Plant J. 20: 629-639.

Potter I and Fry SC (1994). Changes in xyloglucan endotransglycosylase (XET) activity during hormone-induced growth in lettuce and cucumber hypocotyls and spinach cell suspension cultures. J. Exp. Bot. 45: 1703-1710.

Pritchard J, Hetherington PR, Fry SC and Tomos AD (1993). Xyloglucan endotransglycosylase activity, microfibril orientation and the profiles of cell wall properties along growing regions of maize roots. J. Exp. Bot. 44: 1281-1289.

Romo S, Jimenez T, Labrador E and Dopico B (2005). The gene for a xyloglucan endotransglucosylase/hydrolase from Cicer arietinum is strongly expressed in elongating tissues. Plant Physiol. Biochem. 43: 169-176.

Ruan YL and Chourey PS (1998). An altered mode of spatial and temporal expression of sucrose synthase in a fiberless seed mutant of cotton. Plant Physiol. 118: 399-406.

Ruan YL, Llewellyn DJ and Furbank RT (2000). Pathway and control of sucrose import into initiating cotton fibers. Aust. J. Plant Physiol. 27: 795-800.

Ruan YL, Llewellyn DJ and Furbank RT (2001). The control of single-celled cotton fiber elongation by developmentally reversible gating of plasmodesmata and coordinated expression of sucrose and $\mathrm{K}^{+}$transporters and expansin. Plant Cell 13: 47-60.

Ryser U (1999). Cotton Fiber Initiation and Histo Differentiation. In: Cotton Fibers: Developmental Biology, Quality Improvement, and Textile Processing (Basra AS, ed.). Food Products Press, New York, 1-45.

Schubert AM, Benedict CR, Berlin JD and Kohel RJ (1973). Cotton fiber development-kinetics of cell elongation and secondary wall thicking. Crop Sci. 13: 704-709.

Schümann PHD, Smith RC, Lang V, Matthews PR, et al. (1997). Expression of XET-related genes and its relation to elongation in leaves of barley (Hordeum vulgare L.). Plant Cell Environ. 20: 1439-1450.

Shimizu Y, Aotsuka S, Hasegawa O, Kawada T, et al. (1997). Changes in levels of mRNAs for cell wall-related enzymes in growing cotton fiber cells. Plant Cell Physiol. 38: 375-378.

Silva JD, Arrowsmith DA, Hellyer S, Whiteman S, et al. (1994). Xyloglucan endotransglycosylase and plant growth. $J$. Exp. Bot. 45: 1693-1701.

Smart LB, Vojdani F, Maeshima M and Wilkins TA (1998). Genes involved in osmoregulation during turgor-driven cell expansion of developing cotton fibers are differentially regulated. Plant Physiol. 116: 1539-1549.

Sulová Z, Lednicka M and Farkas V (1995). A colorimetric assay for xyloglucan-endotransglycosylase from germinating seeds. Anal. Biochem. 229: 80-85.

Thompson JE, Smith RC and Fry SC (1997). Xyloglucan undergoes interpolymeric transglycosylation during binding to the plant cell wall in vivo: evidence from $13 \mathrm{C} / 3 \mathrm{H}$ dual labelling and isopycnic centrifugation in caesium trifluoroacetate. Biochem. J. 327: 699-708.

Thompson JE and Fry SC (2000). Evidence for covalent linkage between xyloglucan and acidic pectins in suspensioncultured rose cells. Planta 211: 275-286.

Verbelen JP, Vissenberg K, Kerstens S and Le J (2001). Cell expansion in the epidermis: microtubules, cellulose orientation and wall loosening enzymes. J. Plant Physiol. 158: 537-543.

Vissenberg K, Martinez-Vilchez IM, Verbelen JP, Miller JG, et al. (2000). In vivo colocalization of xyloglucan endotransglycosylase activity and its donor substrate in the elongation zone of Arabidopsis roots. Plant Cell 12: 1229-1237.

Vissenberg K, Fry SC and Verbelen JP (2001). Root hair initiation is coupled to a highly localized increase of xyloglucan endotransglycosylase action in Arabidopsis roots. Plant Physiol. 127: 1125-1135.

Vissenberg K, Fry SC, Pauly M, Hofte H, et al. (2005). XTH acts at the microfibril-matrix interface during cell elongation. J. Exp. Bot. 56: 673-683.

Xu Y, Xuede W, Shuli J and Chaohua C (2002). Isolation of high-quality RNA from cotton fiber cells by eliminating the interference of endogenous phenolics and secondary metabolites. Cotton Sci. 14: 143-146.

Zhao PM, Wang LL, Han LB, Wang J, et al. (2010). Proteomic identification of differentially expressed proteins in the Ligon lintless mutant of upland cotton (Gossypium hirsutum L.). J. Proteome Res. 9: 1076-1087. 